\title{
Exact Persistence Exponent for One-dimensional Potts Models with Parallel Dynamics
}

\author{
Gautam I. Menon* and P. Ray ${ }^{\dagger}$ \\ The Institute of Mathematical Sciences, C.I.T. Campus, \\ Taramani, Chennai 600 113, \\ India
}

(November 20, 2018)

\begin{abstract}
We obtain $\theta_{p}(q)=2 \theta_{s}(q)$ for one-dimensional $q$-state ferromagnetic Potts models evolving under parallel dynamics at zero temperature from an initially disordered state, where $\theta_{p}(q)$ is the persistence exponent for parallel dynamics and $\theta_{s}(q)=-\frac{1}{8}+\frac{2}{\pi^{2}}\left[\cos ^{-1}\{(2-q) / q \sqrt{2}\}\right]^{2}[\mathrm{PRL}, \mathbf{7 5}, 751,(1995)]$, the persistence exponent under serial dynamics. This result is a consequence of an exact, albeit non-trivial, mapping of the evolution of configurations of Potts spins under parallel dynamics to the dynamics of two decoupled reaction diffusion systems.
\end{abstract}

PACS:02.50.Ey, 05.40.-a, 05.10.Gg

Typeset using REVTEX

*Email:menon@imsc.ernet.in

†Email:ray@imsc.ernet.in 
Consider a stochastic variable, such as the position of a Brownian particle $x(t)$ moving in one dimension as a function of time $(t)$ or the state $\sigma(t) \quad(+1$ or -1$)$ of an Ising spin evolving under a prescribed probabilistic dynamics. The following question arises naturally in many physical contexts: What is the probability that some attribute of the system, such as the state of the Ising spin or, for the Brownian particle, the sign of its $x$-coordinate, is unchanged in an interval $[0, t]$ ? Such questions relate to the "persistence" properties of stochastic processes and have attracted both theoretical and experimental attention in recent years [1] 3 .

This Letter studies persistence, under parallel dynamics, in one-dimensional ferromagnetic $q$-state Potts models with nearest neighbor interactions, described by the Hamiltonian

$$
H=-\sum_{i} \delta_{\sigma_{i}, \sigma_{i+1}}
$$

where $\sigma_{i}$, the Potts spin at site $i$, takes values $0 \ldots(q-1)$. In terms of the conventional (serial) zero-temperature dynamics of this system, the problem of persistence is posed in the following way: Start from initial configurations in which each spin takes any of the allowed $q$ values with equal probability. Then relax the system by allowing one spin at a time to lower its energy by aligning itself with one of its neighbours, chosen from right or left with equal probability. Such a dynamics is serial (equivalently, sequential) since a single spin update is performed at each step. Now fix any spin and ask: What is the probability, averaged over initial configurations, that this spin has not changed its state up to time $t$ ? This quantity, the persistence probability $P(t)$, was first found numerically to decay as a power law,

$$
P(t) \sim \frac{1}{t^{\theta_{s}(q)}}
$$

with $\theta_{s}(q)$ a new, $q$-dependent non-trivial persistence exponent; the subscript denotes serial dynamics. A subsequent analytic tour de force derived $\theta_{s}(q)$ exactly [2],

$$
\theta_{s}(q)=-\frac{1}{8}+\frac{2}{\pi^{2}}\left[\cos ^{-1}\{(2-q) / q \sqrt{2}\}\right]^{2} .
$$

The persistence exponent is argued to be a new exponent characterizing the dynamics [3] 
Barring this remarkable result, we know of very few other exact results in the persistence problem for many interacting variables. Further, these results, besides being restricted to one dimensional problems, have so far, with the exception of Ref. 四, been confined to serial dynamics. The issue of the dynamics is important here, since the persistence exponent does not share the universal character of the static and dynamic exponents. It is thus of obvious interest to find other exact results for persistence in interacting many-body systems, to understand the origins of such scale invariant behaviour.

This Letter obtains the following result connecting the persistence exponent under parallel dynamics $\theta_{p}(q)$ for one-dimensional $q$-state ferromagnetic Potts models with the corresponding exponent $\theta_{s}(q)$ for serial dynamics:

$$
\theta_{p}(q)=2 \theta_{s}(q),
$$

where $P(t) \sim 1 / t^{\theta_{p}(q)}$ in the parallel case. We demonstrate the validity of this result first numerically and then provide a proof via a sequence of mappings to the dynamics of the following reaction diffusion system:

$$
\begin{array}{rlll}
A+A & \rightarrow \phi & \text { Probability } & 1 /(q-1) \\
\rightarrow A & \text { Probability } & (q-2) /(q-1) \\
B+B \rightarrow \phi & \text { Probability } & 1 /(q-1) \\
\rightarrow B & \text { Probability } & (q-2) /(q-1),
\end{array}
$$

describing particles of two species, labelled $A$ and $B$, which diffuse and interact in one dimension. Apart from the doubling of species, this reaction scheme is identical to the one used in a domain wall mapping for persistence in the Potts model with serial dynamics, with the particles representing the domain walls. The initial distribution of $A$ and $B$ particles can be assigned given any initial configuration of Potts spins through the mapping we describe below. However, as we show here, the $A$ and $B$ particles which enter the analysis of the case of parallel dynamics are not the same as the domain walls in the serial case. 
In terms of this mapping, whether a given site is persistent or not at time $t$ is given by the probability that it is not crossed by either a particle of type A or B upto time $t$. Since the two reactions are independent, provided there are no significant correlations in the initial placement of $\mathrm{A}$ and $\mathrm{B}$ particles, the joint probability that a given site is persistent with respect to the motion of both $\mathrm{A}$ and $\mathrm{B}$ particles simply factors into the product of independent probabilities at long times, as

$$
P(t) \sim \frac{1}{t^{\theta_{s}(q)}} \frac{1}{t^{\theta_{s}(q)}} \sim \frac{1}{t^{2 \theta_{s}(q)}} \sim \frac{1}{t^{\theta_{p}(q)}}
$$

yielding immediately the result of Eq. 4.

We first present results from our numerical simulations of this problem. Each spin can take one of $(q-1)$ values, which we take, for concreteness, to be represented by the integers $\{0 \ldots(q-1)\}$. The zero temperature dynamics evolves a spin configuration $\{\sigma(t)\}$ at time $t$ to a configuration $\{\sigma(t+1)\}$ at time $t+1$ through the following simple rule: Each spin at time $t+1$ assumes the value of one of its neighboring spins at time $t$, chosen from right or left with equal probability. Each such step in time constitutes a single Monte Carlo step. The parallel nature of the dynamics follows from the fact that all spins are updated together in a single time step.

We have simulated parallel dynamics using the above rules on Potts systems with different $q$ values, on lattices of linear size $L=10^{5}$ sites and for times $t \leq 10^{4}$, applying periodic boundary conditions. We average over a fairly large number of initial conditions, typically $10^{2}-10^{3}$, starting from configurations in which each spin is independently assigned a value in the range $\{0 \ldots(q-1)\}$ with equal probability. We compute the standard persistence probability $P(t)$, defined as the probability that the spin at a given site has not changed its state up to time $t$, averaged over all sites and over an ensemble of initial conditions.

Fig. 1 shows the persistence probability $P(t)$ for ferromagnetic Potts systems, evolving under parallel dynamics, for $q=3,5,8$ and 20. Given Eqs. 3 and 4 , we expect a power law tail to $P(t)$ with exponents $\theta_{p}(3) \simeq 1.076, \theta_{p}(5) \simeq 1.386, \theta_{p}(8) \simeq 1.588$ and $\theta_{p}(20) \simeq 1.821$. As can be seen from the fits displayed, the data are consistent with our proposal. 
In a recent paper, coauthored with Shukla [4], we studied the Ising version of the problem of persistence with parallel dynamics, obtaining the result of Eq. H specialized to $q=2$ i.e. $\theta_{p}(2)=3 / 4=2 \theta_{s}(2)=2(3 / 8)$. We briefly sketch the mapping which enabled us to show this exactly and then describe how such a mapping is extended to solve the more general Potts case.

For the one-dimensional Ising model with serial dynamics, domain walls obey the reaction diffusion scheme $A+A \rightarrow 0$. Whether a site is persistent or not at some late time $t$, is equivalent to asking whether it has been crossed by a domain wall at an earlier time. As shown in Ref [四], the diffusing particles whose motion and annihilation govern the persistence properties of the Ising model with parallel dynamics are not the domain walls of the serial case, but entities which we termed as zero-field sites i.e. sites in which the spin is aligned with either one, but not both, of its neighbours.

Given any configuration of the Ising model, all sites can be classified (uniquely) as stable (S), unstable (U) or zero-field (Z) depending on whether they will flip with probability 0,1 or $1 / 2$ respectively in the next time step. While pairs of permanently bound zero-field sites can be identified with the domain walls of the serial case, the two sites constituting such a pair can move independently under parallel dynamics. A region of stable sites is always bounded by zero-field sites; a site embedded in an initially stable region is persistent upto time $t$ if it has not been crossed by a zero-field site in the interval $[0, t]$. The doubling of the persistence exponent is a consequence of the fact that zero-field sites on A and B sublattices decouple from each other under the dynamics. Identifying zero-field sites on the $A$ (or $B$ ) sublattice as particles labelled $A$ or $B$ respectively, the following equations describe the interaction of these particles: $A+A \rightarrow 0$ and $B+B \rightarrow 0$; these equations can be obtained from Eq. 5 upon setting $q$ to 2. As shown in Ref. [1], zero-field sites on the A(B) sublattice at time $t$ hop to sites on the $\mathrm{B}(\mathrm{A})$ sublattice at time $t+1$. The $A$ and $B$ labels are identified with respect to an arbitrary initial time, chosen for convenience to be $t=0$.

It is tempting to conjecture that a similar sublattice decoupling is responsible for the doubling of the persistence exponent with parallel dynamics vis. a vis the serial case, for 
general $q$. This idea, as we will show, is essentially correct, but for the following caveat: The A and B particles are not simply the zero-field sites of the Potts model. This result follows from the observation that the classification of all spins as stable, unstable or zero-field, as outlined in the Ising case, is incomplete when applied to the problem of persistence in Potts models with $q>2$.

Arguments against a naive generalization of ideas from the Ising case follow from inspecting the pictures of Potts configurations evolving under parallel dynamics shown in Figs. 2(a) and 2(b). In these pictures, obtained from simulations of a 3-state Potts model, the configuration corresponding to the earliest time is shown on the top row, while each subsequent row represents the configuration at the following time step. We have identified and labelled zero-field sites in terms of the $A$ and $B$ particles described above. Note that while $A(B)$ particles hop at each time step to sites on the $B(A)$ sublattice in most of the updates shown, this strict independance of the two sublattices is violated in the penultimate row of Fig. 2(a). Evidently, the dynamics of the zero-field sites alone, unlike the case for the one-dimensional Ising model, does not decouple the two sublattices in the more general Potts case. A related difficulty is apparent from inspection of the configurations of Fig. 2(b). Note that an $A B$ pair appears to be created upon evolution. Such a reaction cannot be described by Eq. 5, which yields a strictly non-increasing number of $A$ and $B$ particles.

Our mapping to the reaction diffusion process described by Eq. 5 in terms of configurations of the $q$-state Potts model proceeds as follows: For the purposes of illustration, we consider a 3 -state Potts model in which the states are labelled by 0,1 and 2 . If the spin at site $i$ is in a particular Potts state $q$ while its neighbours are in the same Potts state, for example the central spin in the sequence ...000 .., that site is labelled a stable $(S)$ site. If a given spin at site $i$ has one spin (on either side) in the same state, while the other spin is in a different state, for example the central spin in . .002 .., we will term such a spin as a zero-field $(Z)$ site. As in the Ising case, we will label these zero-field sites as A or B, depending on the sublattice to which they can be assigned. Note that a region of stable sites is bordered by zero-field sites. 
The feature which makes this problem non-trivially different from the Ising case is the following: We must distinguish two categories of unstable sites: unstable sites of type I, labelled $\mathrm{U}_{1}$, have both neighbours in the same state which, however, differs from the state of the central spin. For example, the central spin in the configuration . . 202 ..., is an unstable spin of type I. Finally, we will define unstable sites of type II, labelled $\mathrm{U}_{2}$, in which the spins neighbouring a central spin belong to Potts states which differ from each other as well as from the state of the central spin, the central spin in the configuration $\ldots 012 \ldots$ being an example. Note that unstable sites of type II are absent in the Ising model.

We have now provided a prescription for mapping, given the rules above, any configuration of Potts spins to configurations of $\mathrm{S}, \mathrm{Z}, \mathrm{U}_{1}$ and $\mathrm{U}_{2}$. In the Ising case, $\mathrm{U}_{2}$ sites being absent, we proceeded to identify the zero-field sites $(Z)$ as particles of type $\mathrm{A}$ or $\mathrm{B}$ and went on to show that this mapping yielded precisely Eq. 5 above in the $q=2$ limit, as a consequence of the decoupling of the two sublattices. The central idea which allows us to extend this intuition to the Potts model is the following: We relabel unstable sites of type II, i.e. sites labelled $\mathrm{U}_{2}$, as $\mathrm{A}$ or $\mathrm{B}$ particles, depending on the sublattice to which they belong at $t=0$. Given this relabelling, it is now a trivial, though tedious, exercise to verify that the rules for the interaction of $\mathrm{A}$ and $\mathrm{B}$ particles are precisely those defined by Eq. 5 and that the decoupling of the dynamics of $\mathrm{A}$ and $\mathrm{B}$ particles follows exactly. The chief result of this paper, our Eq. 4, is then a natural consequence of the arguments which led to Eq. 6.

To make this remarkable simplification explicit, we have taken the configurations of Figs. 2(a) and (b) and added the new $A$ and $B$ particles obtained by appropriately labelling unstable sites of type II as described above. These configurations are shown in Figs 3(a) and (b). Note that the incorporation of these new sites immediately solves both problems with the earlier mapping (Figs. 2(a) and (b)) which used only the zero-field sites: The configuration of Fig. 2(a) which showed an A particle remaining on the same sublattice at the following time step is now interpreted in terms of the hopping of both the $A$ particle and an adjacent $B$ particle associated with an unstable site of type II. The difficulty of the apparent creation of an $A B$ pair (Fig. 2(b)), is removed by recognizing that this simply 
arises due to the interconversion of a pair of unstable sites of type II and a pair of zero-field sites.

We now demonstrate, for a simple case, how the rates of Eq. 5 are obtained as a consequence of this mapping. Consider the time evolution of the following spin configuration in a Potts model under parallel dynamics: aaaabccce, where Potts states $a$ and $c$ form stable domains. If $b$ is distinct from both $a$ and $c$ (with $a \neq c$ ), then the mapping described above leads to the following description of the configuration: .......ABA...., where the first zero-field site has been taken to belong, arbitrarily, to sublattice $A$. Note that the two $A$ particles are associated with zero-field sites, whereas the $B$ particle is associated with an unstable site of type II. The following are the possible configurations at the next time step aaaabccc $\rightarrow$ aaaaacccc, aaaacbccc, aaaaabccc, aaaaccccc, aaabacccc, aaabccccc, aaababccc, aaabcbccc. For distinct $a, b$ and $c$, fixing $a$ would give $(q-1)$ choices for $c$ and $(q-2)$ possibilities for $b$, where $q$ is the number of Potts states, yielding a total of $(q-1)(q-2)$ possible variations on $\mathrm{b}$ and $\mathrm{c}$, given $\mathrm{a}$.

Now notice that precisely two of these final states, aaaaacccc and aaaacccce, correspond to the reaction $A+A \rightarrow A$, with the $A$ particle transforming into a $\mathrm{B}$ particle at the next time step; see Fig. 3 for an illustration. All other states can be interpreted in terms of the diffusion of the $A$ and $B$ particles to neighbour sites, keeping in mind the interchange of $\mathrm{A}$ and $\mathrm{B}$ labels at succeeding time steps. Now if $a=c$ while $b \neq a, c$, as in the initial configuration aaaabaaaaa, fixing $a$ would give $(q-1)$ choices for $b$. However, if $a=c$, the spin flip at the "b" site yields, for the final configurations corresponding to the reaction $A+A \rightarrow A$ above, the reaction aaaabaaaaa $\rightarrow$ aaaaaaaaaa, interpreted as $A+A \rightarrow \phi$ in terms of our mapping.

Starting from an initial disordered configuration and averaging over all initial configurations, one would expect configurations of the type (aaaabcccc), with $a \neq b \neq c$, and (aaaabaaaa) with $a \neq b$, to appear in the relative ratio $(q-2)(q-1):(q-1)$. In terms of the dynamics of the $A$ and $B$ particles, this translates into the reaction $A+A \rightarrow A$ occuring with probability $(q-2) /(q-1)$ vis a vis the reaction $A+A \rightarrow \phi$ which occurs with 
probability $1 /(q-1)$. This justifies the rates which appear in our mapping to the system of decoupled reaction diffusion equations illustrated in Eq. 5.

A straightforward corollary to these results is the following: The persistence of a given spin, measured through configurations separated by two time steps, should decay as $P(t) \sim 1 / t^{\theta_{s}(q)}$ i.e. with the serial exponent rather than the parallel one. This is a simple consequence of the fact that only one of the sublattices contributes to persistence in this case. This exact result is interesting in view of recent work on persistence with discrete sampling, in which an underlying continuous time (as opposed to discrete in the case studied here) process is sampled at regular time intervals and its persistence properties studied [5,6].

In conclusion, we have described a mapping between configurations of a one-dimensional $q$-state ferromagnetic Potts model evolving under parallel dynamics and a system of two decoupled reaction-diffusion equations representing the motion and interaction of the boundary sites of stable domains. Using this mapping, we derive a general result connecting persistence exponents obtained with serial and parallel dynamics for this model. This mapping relied on identifying the crucial and somewhat unexpected role of what we have called unstable sites of type II. While the mapping here addressed the specific problem of the persistence exponent, it may be of more general use in the computation of other statistical properties of Potts models evolving under parallel dynamics.

We thank P. Shukla for useful dicussions in the context of Ref [4]. 


\section{REFERENCES}

[1] Derrida B., Bray A.J. and Godre'che C. 1994 J. Phys. A 27 L357

[2] Derrida B., Hakim V. and Pasquier V. 1995 Phys. Rev. Lett 75751

[3] For a review, see Majumdar S.N. 1999 Curr. Sci 77370

[4] Menon G. I., Ray P. and Shukla P. 2001 Phys. Rev. E 64046102

[5] Majumdar S.N., Bray A.J. and Ehrhardt G.C.M.A. 2001 Phys. Rev. E 64 015101(R)

[6] Majumdar S.N. and Dhar D. 2001 Phys. Rev. E 64046123 


\section{FIGURES}

FIG. 1. The persistence probability $P(t)$ in $1 \mathrm{~d}$ ferromagnetic $q$ - state Potts models evolving from random configurations, plotted against time $t$ in a logarithmic scale, for $\mathrm{q}=3,5,8$ and 20 . The dynamics is parallel; all spins are updated in a single time step. The lines represent fits with

exponents $\theta_{p}(3) \simeq 1.076, \theta_{p}(5) \simeq 1.386, \theta_{p}(8) \simeq 1.588$ and $\theta_{p}(20) \simeq 1.821$ [see text].

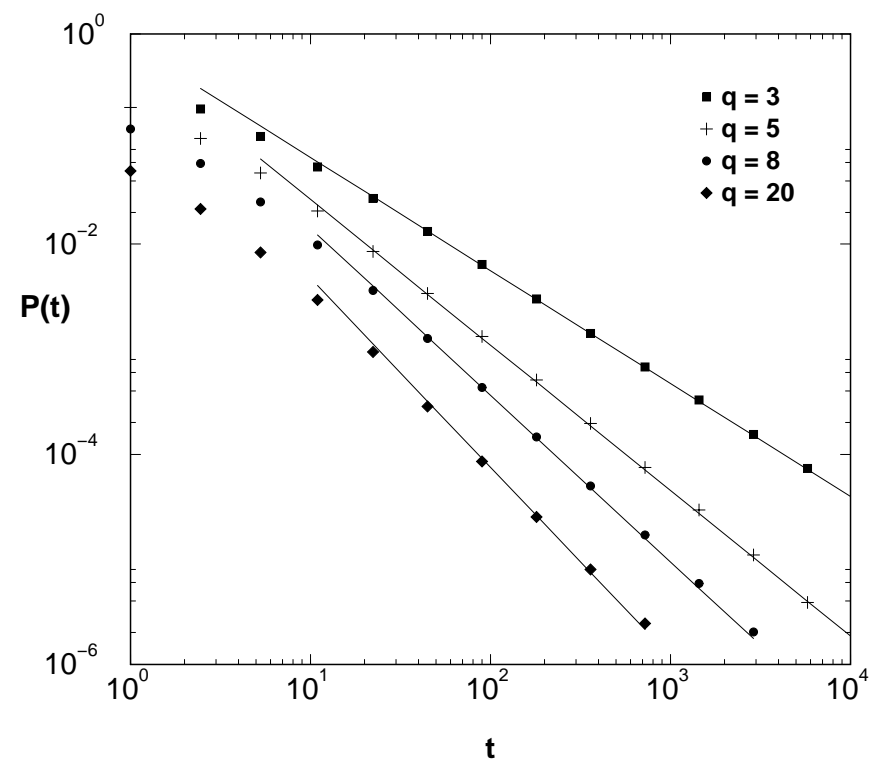


FIG. 2. Evolution of configurations in a 3-state Potts model, in which only zero-field sites (see text) are identified and labelled by the sublattice to which they belong. The configurations are separated by a time step; earlier times appear to the top of the figure. Fig. 2(a) illustrates how the alternation of sublattices at succeeding time steps appears to be violated while 2(b) illustrates how zero-field sites can be destroyed as well as created, in contrast to the results of a similar mapping for the Ising model.

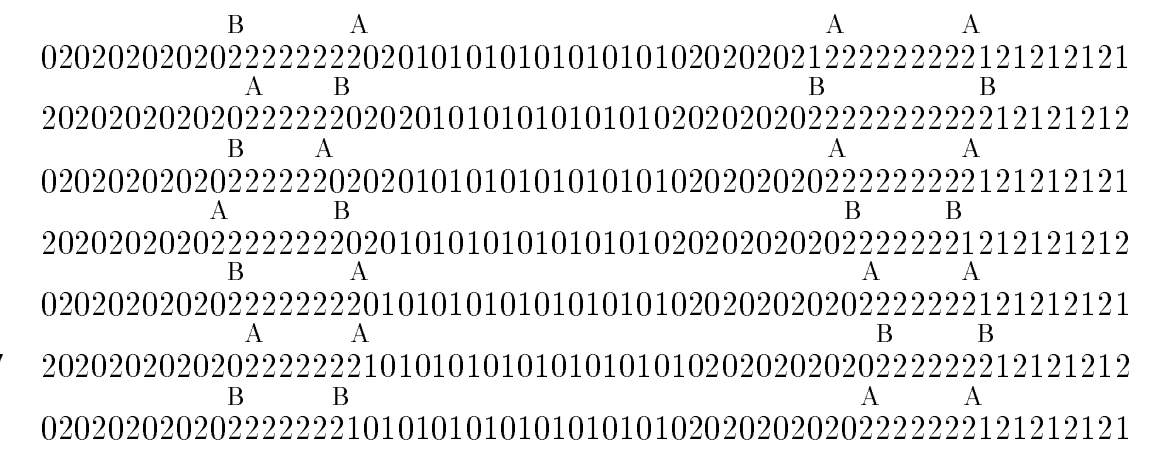

(a)

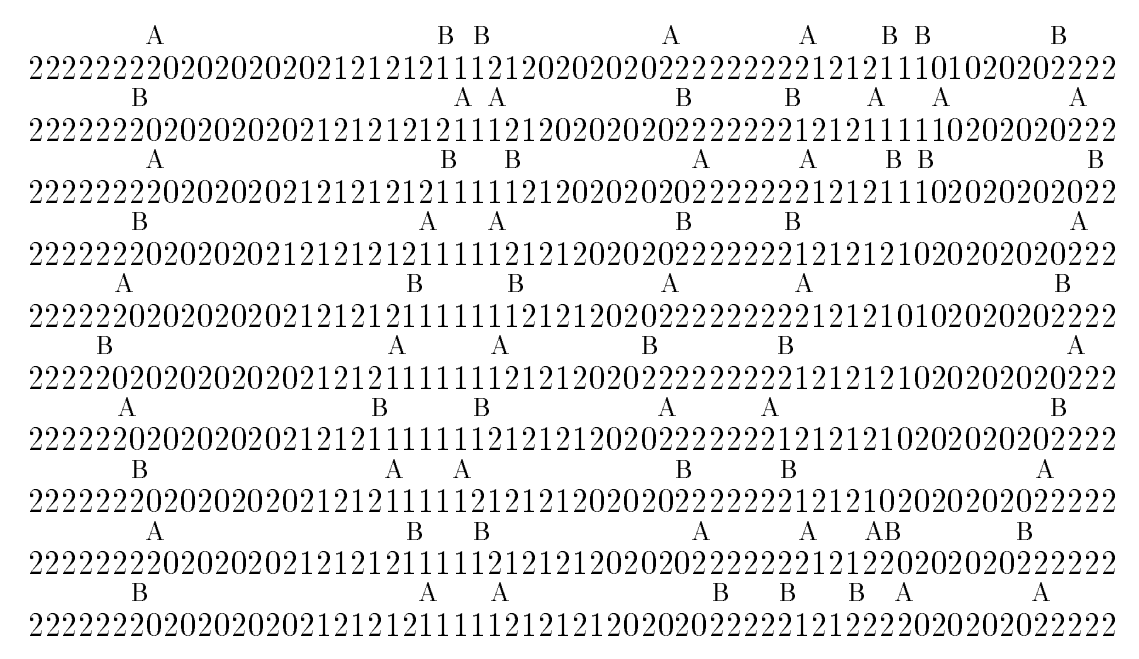

(b) 
FIG. 3. Evolution of the configurations of the 3-state Potts model illustrated in Fig 2, in which unstable sites of type-II (see text) in addition to zero-field sites are identified and labelled according to the sublattice on which they are present. The configurations are separated by a time step; earlier times appear to the top of the figure. The reaction scheme discussed in the text is precisely followed upon the inclusion of unstable sites of type II. Note that the two-sublattice decoupling is an exact feature of the model in which both zero-field sites as well as unstable sites of type-II are identified with the diffusing and annihilating random walkers of the reaction diffusion scheme outlined in the text.

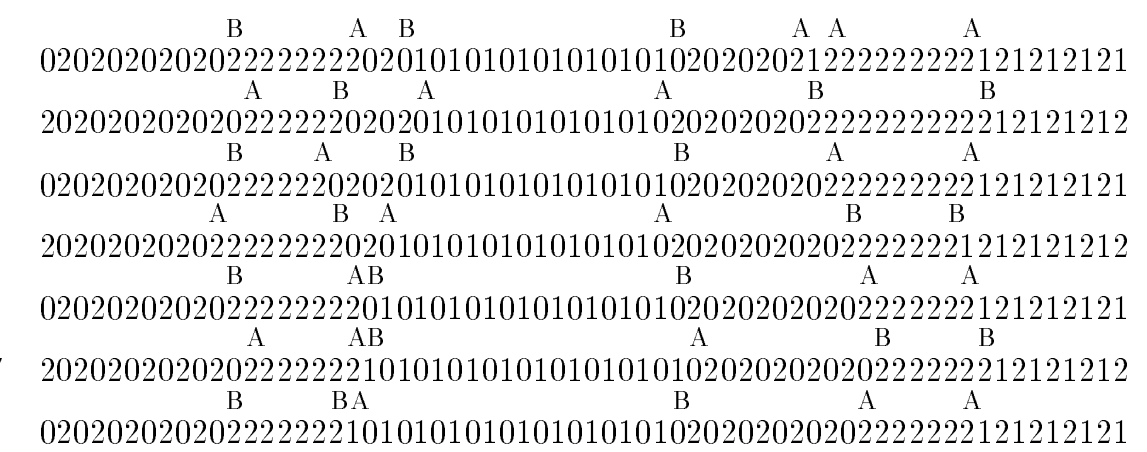

(a)

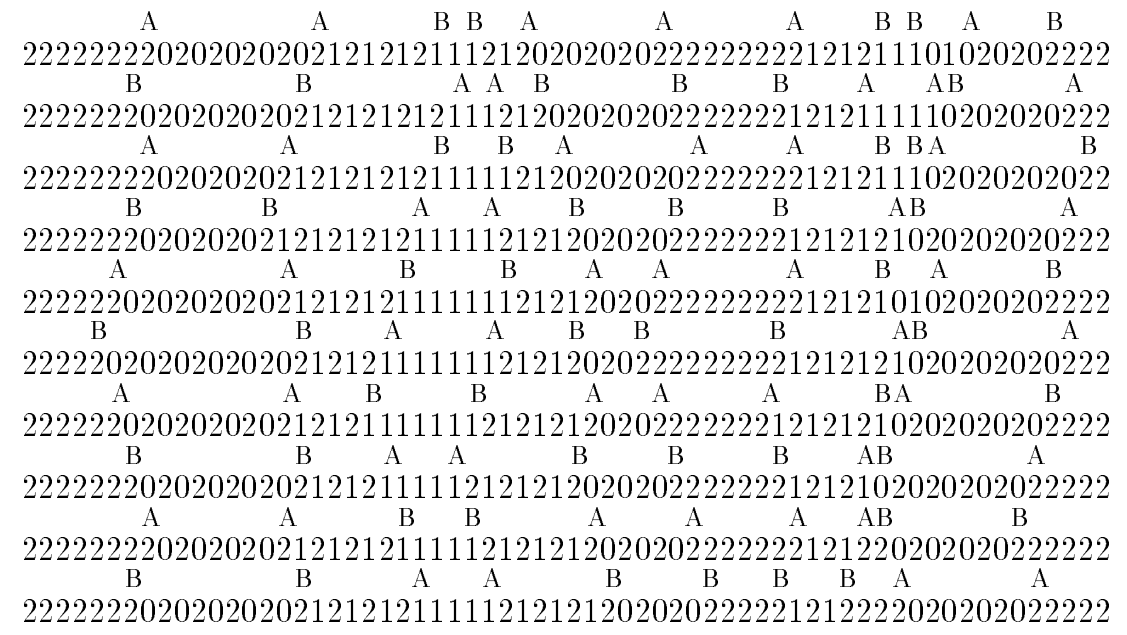

(b) 